\title{
Status and prospects of the Hyper-Kamiokande project
}

\section{Yoshitaka Itow for the Hyper-Kamiokande proto-collaboration ${ }^{*}$}

Institute for Space-Earth Environmental Research / Kobayashi-Maskawa Institute for the Origin of Particles and the Universe, Nagoya University, Nagoya, Aichi, Japan

E-mail: itowdisee.nagoya-u.ac.jp

The Hyper-Kamiokande project is a next-generation gigantic water Cherenkov detector at Kamioka together with a high-intensity 1.3 MW neutrino beam from J-PARC and the Near Detector complex. The project will substantially enhance all currently ongoing physics programs at Super-Kamiokande and $\mathrm{T} 2 \mathrm{~K}$ such as the precise measurement of neutrino oscillations and its $\mathrm{CP}$-asymmetry, low energy neutrino astronomy by solar and supernova neutrinos, search for nucleon decay and for possible other new phenomena. Here, we will present a recent update on the project status and milestones toward the commissioning of the detector foreseen in 2027, and its improved sensitivity, mainly focusing on neutrino oscillations and the search for nucleon decay.

36th International Cosmic Ray Conference -ICRC2019-

July 24th - August 1st, 2019

Madison, WI, U.S.A.

${ }^{*}$ For collaboration list see PoS(ICRC2019)1177

${ }^{\dagger}$ Speaker. 


\section{Introduction}

Neutrino physics has greatly progressed in recent several decades by massive active detectors utilizing the ring imaging water Cherenkov technique. The detectors were originally built motivated by nucleon decay searches. Since atmospheric neutrino oscillations were discovered by Super-Kamiokande [四, a picture of neutrino oscillations in 3-flavor mixing have been intensively studied by various experiments with atmospheric, solar [ [Q, B], reactor [四], and accelerator-based neutrinos. The remaining unknown parameters so far are the CP-phase angle $\delta_{C P}$, the octant of $\theta_{23}$ and the mass ordering between $\mathrm{m}_{2}$ and $\mathrm{m}_{3}$. Recent results for $v_{e}$ appearance measurements by the Tokai-to-Kamioka (T2K) experiment [可], together with the precision measurements of $\theta_{13}$ by reactor neutrino experiments [ 6 ], have already begun to exclude the absence of $\mathrm{CP}$ violation in the neutrino oscillations. A further high-precision neutrino oscillation experiment with a high intensity MW-class neutrino beam is indispensable to tackle the problem.

The Hyper-Kamiokande project is a next-generation gigantic water Cherenkov detector at Kamioka together with a high-intensity neutrino beam from the Japan Proton Accelerator Research complex (J-PARC) at Tokai, Ibaraki Prefecture, and the Near Detector complex in order to pursue high-precision neutrino physics and nucleon decay searches. The project will substantially enhance every physics program currently ongoing in Super-Kamiokande and T2K, such as precise measurement of neutrino oscillations and their CP-asymmetry, low energy neutrino astronomy in solar and supernova neutrinos, the search for nucleon decay, and for possible other new phenomena. A detaied description of the detectors and the projected physics sensitivities can be found elsewhere [ర]].

We will present here a recent update on the project status and milestones toward the commissioning of the detector foreseen in 2027 , and its improved sensitivity, mainly focusing on neutrino oscillations and the search for nucleon decay and other exotic phenomena.

\section{The Hyper-Kamiokande project}

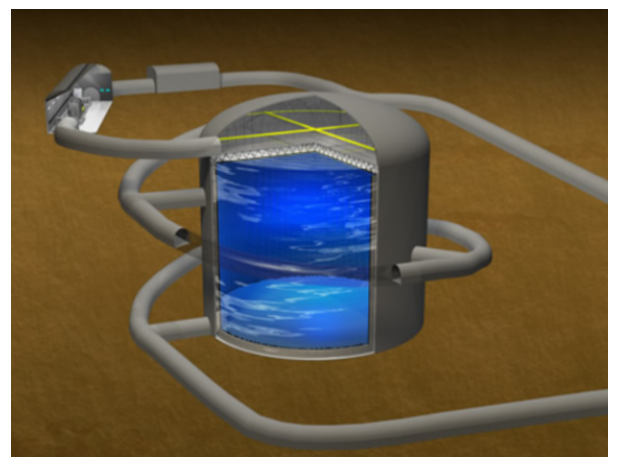

Figure 1: Hyper-Kamiokande detector. The cylindrical tank with a diameter of $74 \mathrm{~m}$ and a height of $60 \mathrm{~m}$ is filled with $258 \mathrm{kt}$ of pure water. 


\subsection{The Hyper-Kamiokande detector}

The experimental setup of the Hyper-Kamiokande project consists of the J-PARC neutrino beam line, the Near Detector complex, and the Hyper-Kamiokande detector as the Far Detector and as a multi-purpose detector.

The Hyper-Kamiokande detector is planned to have a cylindrical tank with a diameter of $74 \mathrm{~m}$ and a height of $60 \mathrm{~m}$. The detector cavity is to be excavated in Mt. Nijugo-yama in the Tochiboramine in the Gifu Prefecture which is approximately $8 \mathrm{~km}$ south of the Super-Kamiokande site. At this location, a neutrino beam from J-PARC will be exposed with the same off-axis angle as that of Super-Kamiokande in the T2K experiment. The rock overburden of the site is approximately 650 $\mathrm{m}$, corresponding to $1750 \mathrm{~m}$ water-equivalent. The cavity is planned to be covered with a highdensity polyethylene lining, and will be filled-up with a $258 \mathrm{kt}$ of the ultra-pure water, providing a fiducial volume of $186 \mathrm{kt}$. The detector is separated into the inner-detector and the outer-detector with a thickness of 1-1.5 m. Forty percent of the inner surface of the inner-detector will be covered by photo-sensors with roughly two times the photo-detection efficiency. Therefore the expected Cherenkov photon yields can be double that of Super-Kamiokande. There have been many R\&D efforts for photo-sensors dedicated to the Hyper-Kamiokande detector. In the current baseline design, newly developed high-efficiency and high-resolution 20-inch PMTs (HAMAMATSU R12860) as well as other concepts of PMTs, such as a multi-PMT, will be primarily deployed.

This configuration can provide a state-of-art of Cherenkov ring reconstruction of the events wtih a threshold as low as a few $\mathrm{MeV}$. The neutron-tagging technique of detecting a $2.2 \mathrm{MeV} \gamma$ from hydrogen capture, which has been successfully developed in Super-Kamiokande, will play an important role in Hyper-Kamiokande physics programs. Selection of $\overline{v_{e}}$ charged current (CC) events by neutron tagging through $\overline{v_{e}}+p \rightarrow e^{+}+n$ is essential for super-nova neutrino physics. Neutron detection is also useful to distinguish the atmospheric neutrino background in proton decay analysis or to improve the reconstruction of neutrino interaction in oscillation physics. Enhancement of neutron detection by loading Gd-sulfate is foreseen in Super-Kamiokande. The feasibility of loading Gd-sulfate in Hyper-Kamiokande is also being considered as a possible future extension.

\subsection{J-PARC neutrino beamline and long baseline program}

The neutrino beam produced at J-PARC, which currently serves the T2K experiment, will be also used for the long-baseline neutrino-oscillation program from Tokai to Hyper-Kamiokande (T2HK). The 30-GeV protons extracted from the Main Ring (MR) collide with a 91-cm long graphite target followed by three sets of horn magnets that select the sign of secondary pions and focus them in a direction aimed $2.5^{\circ}$ away from Super-Kamiokande and Hyper-Kamiokande. This off-axis neutrino-beam configuration can provide an intense narrow band neutrino beam peaked at $600 \mathrm{MeV} / c$ at the first oscillation maximum for $v_{\mu}-v_{\tau}$ oscillations for the $295 \mathrm{~km}$ baseline between Tokai and Kamioka. So far a beam intensity of $2.45 \times 10^{14}$ protons-per-pulse (ppp) corresponding to approximately $485 \mathrm{~kW}$ beam power is achieved. Aiming to the high intensity neutrino beam that is necessary for T2HK, a plan for a beam power upgrade up to $1.3 \mathrm{MW}$ is now being pursued by increasing the beam repetition rate and ppp.

The produced neutrino beam is measured for the flux, energy spectrum, direction, and neutrino component, etc., by the Near Detector complex at the Tokai site to give an essential input for oscil- 
lation analysis. In addition to the idea of re-using the current T2K ND280 detector and its upgrade detectors, which are planned for construction by 2021, a new water Cherenkov detector at about $1-2 \mathrm{~km}$ distance (the Intermediate Water Cherenkov Detector) has been proposed for studying neutrino interactions at the water target with the same technique as used in Super-Kamiokande. This idea proposes to excavate a long vertical pit of $10 \mathrm{~m}$ diameter and $50 \mathrm{~m}$ deep filled with water. A $10-\mathrm{m}$ tall detector structure instrumented with mPMTs inward can move inside the water pit to measure the interaction of various neutrino spectra at different off-axis angles from $1^{\circ}$ to $4^{\circ}$. This " $v$ Prism" idea can improve knowledge on energy-dependent neutrino interactions, which is essential to achieve the ultimate goal of reducing systematic errors for the oscillation analysis in T2HK.

\section{Physics in Hyper-Kamiokande}

\subsection{Neutrino oscillation physics}
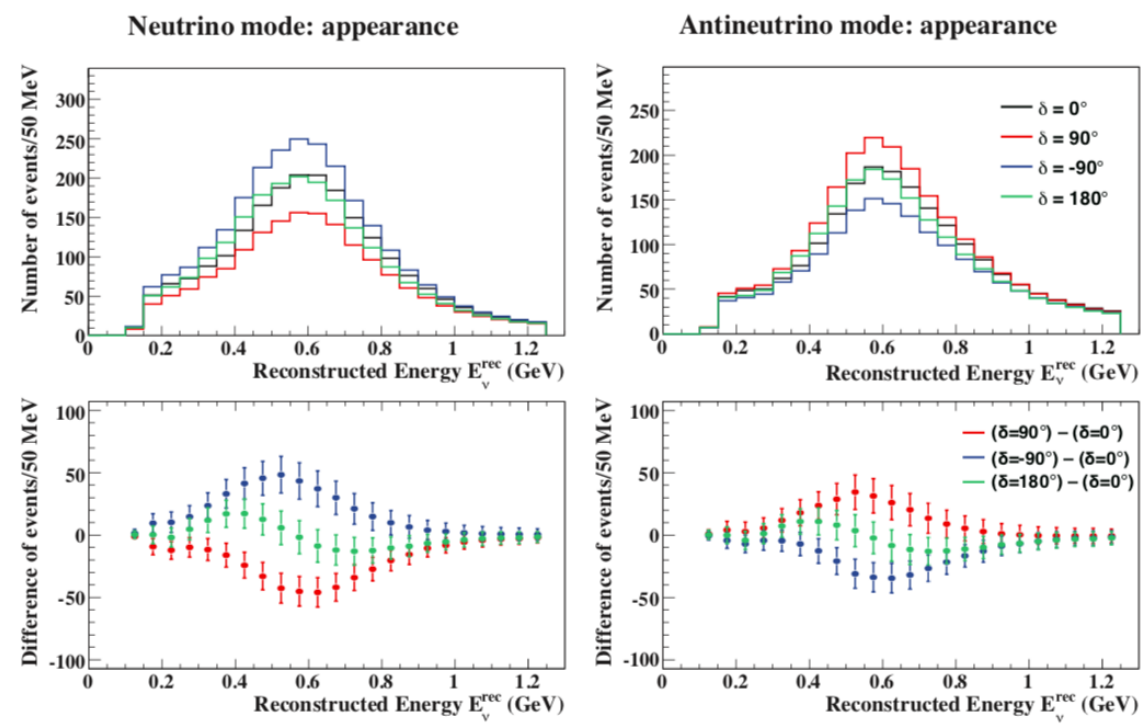

Figure 2: Reconstructed energy distributions of the $v_{e}$ events for each $\delta_{C P}$ value for neutrino mode (left) and antineutrino mode (right). Assumes $\sin ^{2} 2 \theta_{13}=0.1$ and normal hierarchy. Bottom plots show the difference of the reconstructed energy distributions from the case where $\delta_{C P}=0^{\circ}$.

A long-baseline neutrino-oscillation program is one of the key elements of the Hyper-Kamiokande project. A low-energy narrow-band neutrino beam designed to be at oscillation maximum is suitable to measure $v_{e}$ appearance. At the peak neutrino energy around $600 \mathrm{MeV}, \mathrm{CC}$ quasi-elastic (QE) interaction dominates so that neutrino energy can be clearly reconstructed. The matter oscillation effect is suppressed for a relatively short baseline of $295 \mathrm{~km}$, which is suitable to extract $\delta_{C P}$ information. Figure 1 . ل shows the reconstructed energy distributions of the $v_{e}$ events for each $\delta_{C P}$ value for $v_{\mu}-v_{e}$ (left) and $\overline{v_{\mu}}-\overline{v_{e}}$ (right). As shown in Fig. B.], direct comparison of $v_{\mu}-v_{e}$ and $\overline{v_{\mu}}-\overline{v_{e}}$ will bring clear model-independent evidence of $\mathrm{CP}$ violation in neutrino oscillations. The 
expected significance to exclude $\sin \delta_{C P}=0$ as a function of true $\delta_{C P}$ is shown in Fig. B.] ( left ) for a 10 -year run at $1.3 \mathrm{MW}$. For this case $\sin ^{2} 2 \theta_{13}=0.1$ and $\sin ^{2} \theta_{23}=0.5$ are assumed. If the true $\delta_{C P}$ is near the value that the current $\mathrm{T} 2 \mathrm{~K}$ result suggests, a non-zero $\sin \delta_{C P}$ will be rejected by almost $8 \sigma$, and $5 \sigma$ can be established even within a few years of operation. Fig. B.] ( right ) shows an expected $68 \% \mathrm{CL}$ uncertainty of $\delta_{C P}=0$ as a function of running time. Here, we assume one year is $10^{7} \mathrm{~s}$ and the mass hierarchy is known.
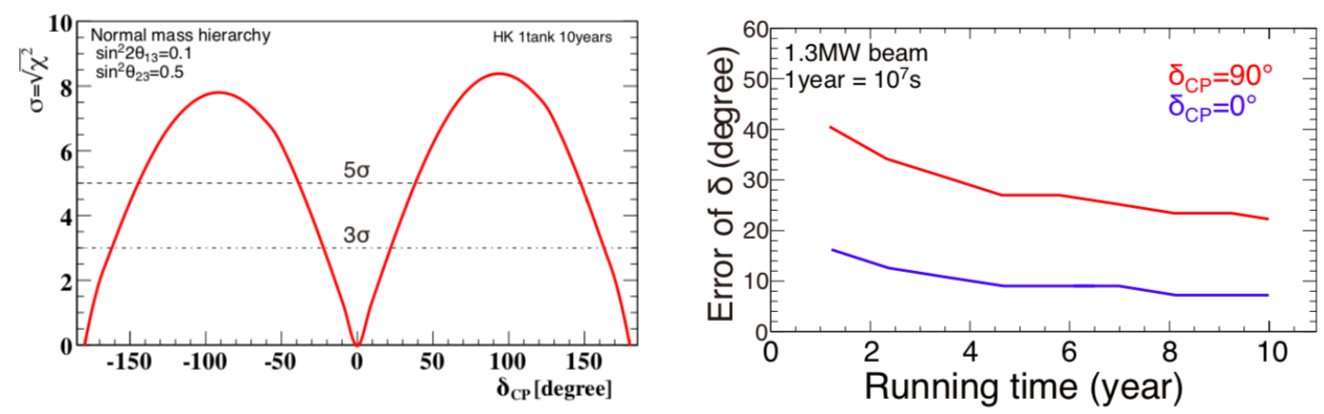

Figure 3: Projected significance for each $\delta_{C P}$ value for T2HK 10 years run (left). Accuracy of $\delta_{C R}$ as a function of time in T2HK (right).

Measurement of atmospheric neutrinos is another important program for precision study of neutrino oscillation in Hyper-Kamiokande, thanks to unique advantages such as a wide energy coverage, long travel distance through the earth, and free running. Atmospheric neutrino measurement is sensitive to mass ordering or the octant of $\theta_{23}$ which are complementary results to the long baseline program. Figure 3.1 shows the sensitivity for wrong mass hierarchy rejection by atmospheric neutrino analysis combined with the T2HK results over 10 years. Irrelevant to an assumption of mass hierarchy, the combined analysis of atmospheric neutrinos and long baseline neutrinos can reject the wrong assumption by a significance better than $3 \sigma$ even for the case of $\sin ^{2} \theta_{23}=0.4$.

There have been discussions of building another large water Cherenkov detector at the southern part of the Korean peninsula [ [8]. That site can be exposed by a J-PARC neutrino beam with $1-3^{\circ}$ off-axis at a baseline of $1000-1300 \mathrm{~km}$, and the measurement can be eventually realized at the second oscillation maximum where a large probability difference between neutrino and anti-neutrino oscillations can be expected. The combination of two water Cherenkov detector measurements at different baselines ( $\mathrm{L}=295 \mathrm{~km}$ and $\mathrm{L}=1100 \mathrm{~km}$ ) provides a unique method with less systematics to break the degeneracy in oscillation parameters and to explore possible effects beyond the 3-flavor mixing scheme. This idea, namely T2HKK or Korean Neutrino Observatory (KNO), is intensively discussed as an attractive future extension after the first cavity is realized.

\subsection{Nucleon decay searches}

Gigantic water Cherenkov detectors such as Kamiokande or IMB were originally constructed to search for nucleon decays. So far there has been no evidence reported and upper limits for various decay modes are available. Super-Kamiokande has reported no positive signals for two well-known decay modes $p \rightarrow e^{+} \pi^{0}$ and $p \rightarrow \bar{v}+K^{+}$, giving the current best upper limits as $2.0 \times 10^{34}$ years 


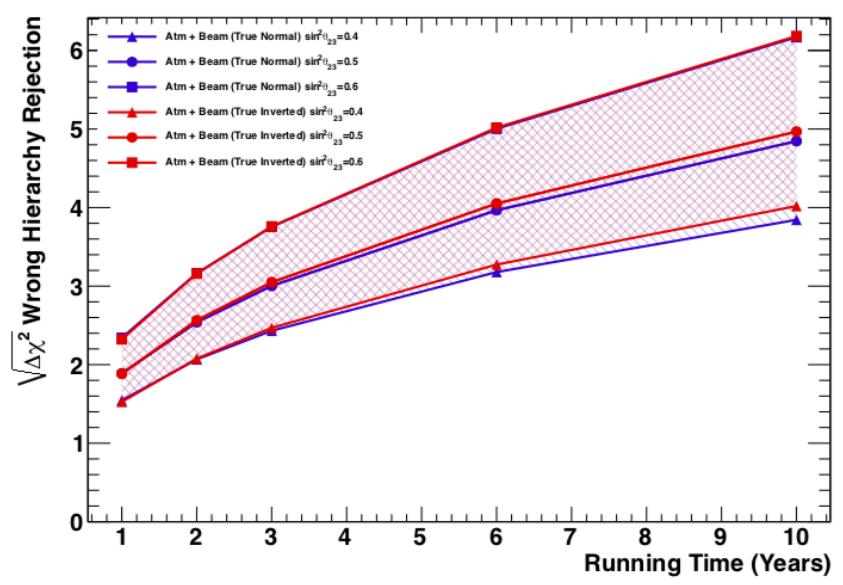

Figure 4: Projected sensitivity for wrong mass hierarchy rejection by atmospheric neutrino analysis combined with accelerator neutrinos. For different $\theta_{23}$ octant assumptions; $\sin ^{2} \theta_{23}=0.4$ (triangle), $\sin ^{2} \theta_{23}=$ 0.5 (circle), and $\sin ^{2} \theta_{23}=0.6$ (square). Blue and red colors indicate normal and inverted hierarchy for true cases.

and $6.6 \times 10^{33}$ years $(90 \% \mathrm{CL}, 365 \mathrm{kt}$ years). The Hyper-Kamiokande will greatly improve the sensitivity thanks to the eight times larger fiducial mass than that of Super-Kamiokande. Moreover, Hyper-Kamiokande can provide concrete evidence for nucleon decay if it happens. It becomes more important to discriminate background events due to atmospheric neutrino interactions for such a large exposure. A clear signal of a $p \rightarrow e^{+} \pi^{0}$ event is back-to-back three Cherenkov rings from an $e^{+}$and two $\gamma$-rays from a $\pi^{0}$ decay, where the reconstructed mass and the momentum balance of three rings are consistent with the assumption for the decay of a proton at rest. Figure B.2 shows kinematic distributions for $p \rightarrow e^{+} \pi^{0}$ and atmospheric neutrino background events. We would see a clear peak in the reconstructed mass, which is consistent with a free proton decay, especially in the events where total momentum is nearly zero. Another important technique is neutron tagging because neutrino interactions would likely produce neutrons in their final states, whereas nucleon decay events may not. As for the $p \rightarrow \bar{v}+K^{+}$, a key of the analysis is detection of a $6 \mathrm{MeV}$ de-excitation $\gamma$-ray from a ${ }^{15} N$ hole state of after the proton decays. Thanks to the high efficiency and high resolution of new PMT's, approximately 12 hits per MeV are expected with the $40 \%$ photo-coverage. Therefore, sensitivity for $6 \mathrm{MeV} \gamma$-rays from $p \rightarrow \bar{v}+K^{+}$should be improved. Figure 3.2 shows the projected $3 \sigma$ sensitivity for the life-time as a function of observation time for $p \rightarrow e^{+} \pi^{0}$ (left) and for $p \rightarrow \bar{v}+K^{+}$(right). Hyper-Kamiokande will improve the current sensitivity of Super-Kamiokande in a few years and will reach a new region of $10^{35}$ years lifetime for the first for $p \rightarrow e^{+} \pi^{0}$ modes.

\subsection{Exotic searches and other physics programs}

The Hyper-Kamiokande is a multi-disciplinary project having a variety of cross-cutting topics. Precise observation of solar neutrinos is one of the most important items of Hyper-Kamiokande physics for precision neutrino oscillation study with $\Delta m_{21}^{2}$ and also for solar physics. Low-energy $\mathrm{MeV}$ astrophysics such as supernova and diffuse supernova neutrino background is another im- 

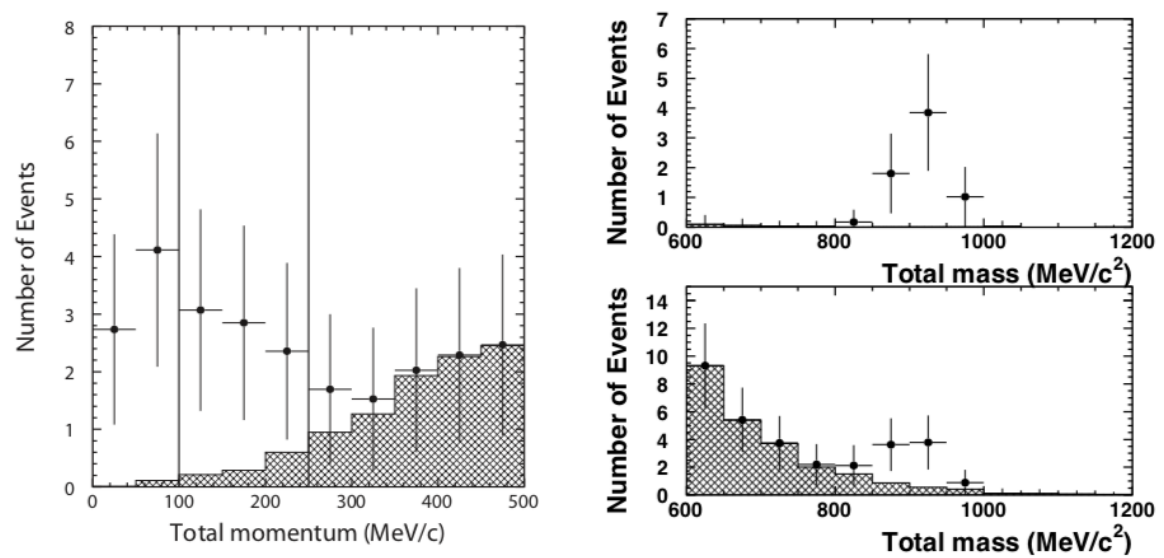

Figure 5: Expected distributions for $p \rightarrow e^{+} \pi^{0}$ proton decay analysis. Total momentum distribution of the events (left). Reconstruction mass of the events for free-protons where total momentum $<100 \mathrm{MeV} / c$ (top right) and bounded-protons where $100 \mathrm{MeV} / c<$ total momentum $<250 \mathrm{MeV} / c$ (bottom right).
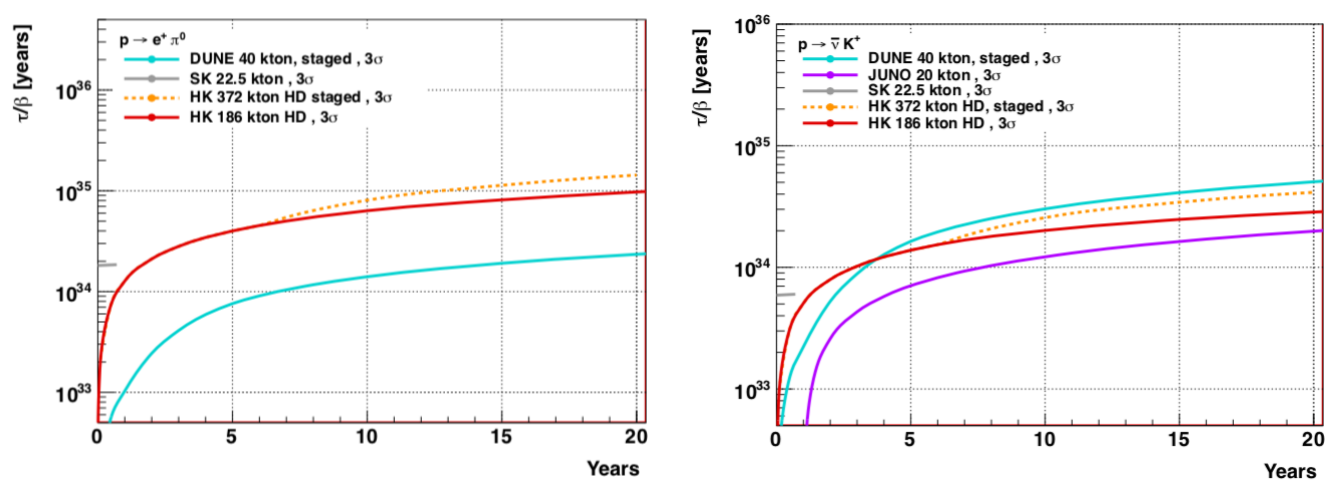

Figure 6: Projected $3 \sigma$ discovery sensitivity as a function of observation time for $p \rightarrow e^{+} \pi^{0}$ (left) and $p \rightarrow \bar{v}+K^{+}$(right).

portant topic in which Hyper-Kamiokande can play a unique role. These topics in low-energy astrophysics are discussed elsewhere.

As for synergy with the neutrino telescopes such as IceCube or KM3NeT, GeV-energy astrophysics would be an interesting topic in which Hyper-Kamiokande can take a unique role. In the $\mathrm{GeV}$ range, Super-Kamiokande has established excellent Cherenkov ring reconstruction and precise modeling of atmospheric neutrino oscillations, which are also useful to astrophysical neutrino searches. Coincidence searches of transient astronomical objects, such as gravitational wave alerts, would be one example. Another important topic would be indirect dark matter searches using neutrinos, such as the solar WIMP search. This method is unique and useful to explore the spin-dependent cross section for light WIMPs. Hyper-Kamiokande can improve current limits by Super-Kamiokande by a factor of at least $3-4$ by improving statistics. There is also discussion on an idea for neutrino tomography of the earth discussed by using precision measurement of matter 
effects in atmospheric neutrino oscillations. There is a variety of physics cases foreseen in the Hyper-Kamiokande project covering many different research fields.

\section{Conclusion and future prospects}

Neutrinos and nucleon decays are important research topics. In addition, the $\mathrm{MeV}-\mathrm{GeV}$ neutrino is a unique and important probe for exploring astroparticle physics in the multi-messenger era. A water Cherenkov detector has a long history and is a well-established technology suitable for these purposes.

Aiming to start operation from 2027, initial construction work is foreseen in 2020. Based on the legacy of water Cherenkov detectors as well as the successful experience of long baseline neutrino experiments, the Hyper-Kamiokande project will take on the role of a next-generation neutrino and nucleon decay experiment.

\section{References}

[1] Y. Fukuda et al. (Super-Kamiokande), Phys. Rev. Lett. 81, 1562 (1998), arXiv:hep-ex/9807003.

[2] K. Abe et al. (Super-Kamiokande), Phys. Rev. D83, 052010 (2011), arXiv:1010.0118 [hep-ex].

[3] Q. R. Ahmad et al. (SNO), Phys. Rev. Lett. 89, 011301 (2002), arXiv:nucl-ex/0204008 [nucl-ex].

[4] K. Eguchi et al. (KamLAND), Phys. Rev. Lett. 90, 021802 (2003), arXiv:hep-ex/0212021 [hep-ex].

[5] K. Abe et al. (T2K), Phys. Rev. D85, 031103 (2012), arXiv:1201.1386 [hep-ex].

[6] F. P. An et al. (Daya Bay), Phys. Rev. Lett. 108, 171803 (2012), arXiv:1203.1669 [hep-ex].

[7] K. Abe et al. [Hyper-Kamiokande Collaboration], arXiv:1805.04163 [physics.ins-det].

[8] S. H. Seo, arXiv:1811.06682 [hep-ex]. 